\title{
Driving Forces of Individual Investors in Stock Market Participation
}

\author{
Candauda Arachchige Saliya*
}

College of Business, Fiji National University, P.O. Box 7222, Nasinu, Fiji.

\begin{abstract}
The purpose of this study is to estimate the impact of sociocultural factors on investors' behavior in the South Pacific Stock Market (SPX) participation in Fiji using a novel methodological framework. We constructed a higher order factor: individual investors' willingness to participate in the SPX by confirming second-order Factor Structure and Overall Model Fit of the underpinning socioeconomic, cultural and psychic motivating factors thereof. The theoretical framework is primarily based on the Theory of Planned Behavior (TPB). Four dimensions were hypothesized: Attitudes, Perceived Power, Social Norms, and Financial Knowledge, and showed significant and substantial loadings towards the investment willingness, with an acceptable model fit. The results confirm that four hypothesized dimensions are identifiable and distinct aspects; Social Norms dimension showed significant positive association $(\beta=.159, \mathrm{p}<.05)$ while, more importantly, the Perceived Power dimension showed a highly significant negative association $(\beta=-0.246, p<.01)$ with investment activities of the participants. These findings may be useful in designing policies and strategies for stimulating the financial market in Fiji, especially, the SPX.
\end{abstract}

Keywords: decision making; financial markets; investment culture; judgement; socio-economic environment.

JEL classification: B41- Economic Methodology; B26 - Financial Economics; G41- Role and Effects of Psychological, Emotional, Social, and Cognitive Factors on Decision Making in Financial Markets (Neurofinance).

\section{INTRODUCTION}

There are many reasons for an increase in stock market investments-financial market assets' profitability, flexibility and liquidity and diversity - which means that investors can find assets that suit their investment objectives. However, the underpinning driving forces of investor motivation could vary from person to person, from culture to culture, as we see diverse developments in stock markets amongst various countries. Purpose of this study is to estimate the impact of such sociocultural factors on investors' behavior in the South Pacific Stock Market (SPX) participation in Fiji using a novel methodological framework based on The Theory of Planned Behavior (TPB).

TPB is one of commonly used models explaining informational and motivational influences on human behavior (Ajzen 1991). The theory defines behavior in terms of three dimensions (attitude, subjective norms and perceived behavioral control), that capture behavioral intentions. Attitude can be defined as the degree to which an individual derives positive or negative valuation from performing a specific behavior (Ajzen, 1991), whereas subjective norms are defined as social pressures that compel an individual to engage in a specific behavior (Ajzen 1991). Behavioral control measures the perceived ease or difficulty that an individual faces while performing certain behaviors (Ajzen 1991).

In addition, many researchers recognize financial knowledge as a vital dimension in determining financial behavior (Khan

*Address correspondence to this author at College of Business, Fiji National University, P.O. Box 7222, Nasinu, Fiji. Mobile: +642041148029; Res: +6495804078; E-mail: saliya.ca@gmail.com
2016; Tauni, Fang and Iqbal 2017; Akhtar and Das 2019). Therefore, it was added to the TPB framework as the fourth influential dimension to develop this methodology design and model.

\section{THEORY}

Information costs can be a significant barrier of entry in the stock markets, and low cognitive abilities are likely to further increase these costs (Gumbo and Sandada 2018). Benjamin, Brown, and Shapiro (2006) find that more cognitively able individuals are more risk-neutral and cognitively gifted individuals are less likely to display behaviors associated with high risk aversion such as financial market participation (Gumbo and Sandada 2018). Benjamin, Brown, and Shapiro (2006) counter-argue that greater cognitive skills lead to improved reasoned financial choices and a higher likelihood of stock market participation-it may also be conceivable that low cognitive skills increase stock market participation. Korniotis and Kumar (2011) find a relationship between low cognitive ability and overconfidence.

Investors who perceive high levels of uncertainty are more likely not to participate in the stock market (Makarov and Schornick 2010). Stock market participation is an important economic outcome. There can be a substantial welfare loss from not participating in the stock market, as exposure to equities, and hence to the equity premium, may be an important determinant of the long-run return to individual savings (Cocco and Gomes 2012). Individuals with higher levels of self-efficacy, a cumulative set of skills and strengths, perceive themselves as able to examine, process and make correct inferences from limited and unclear information (Cho 


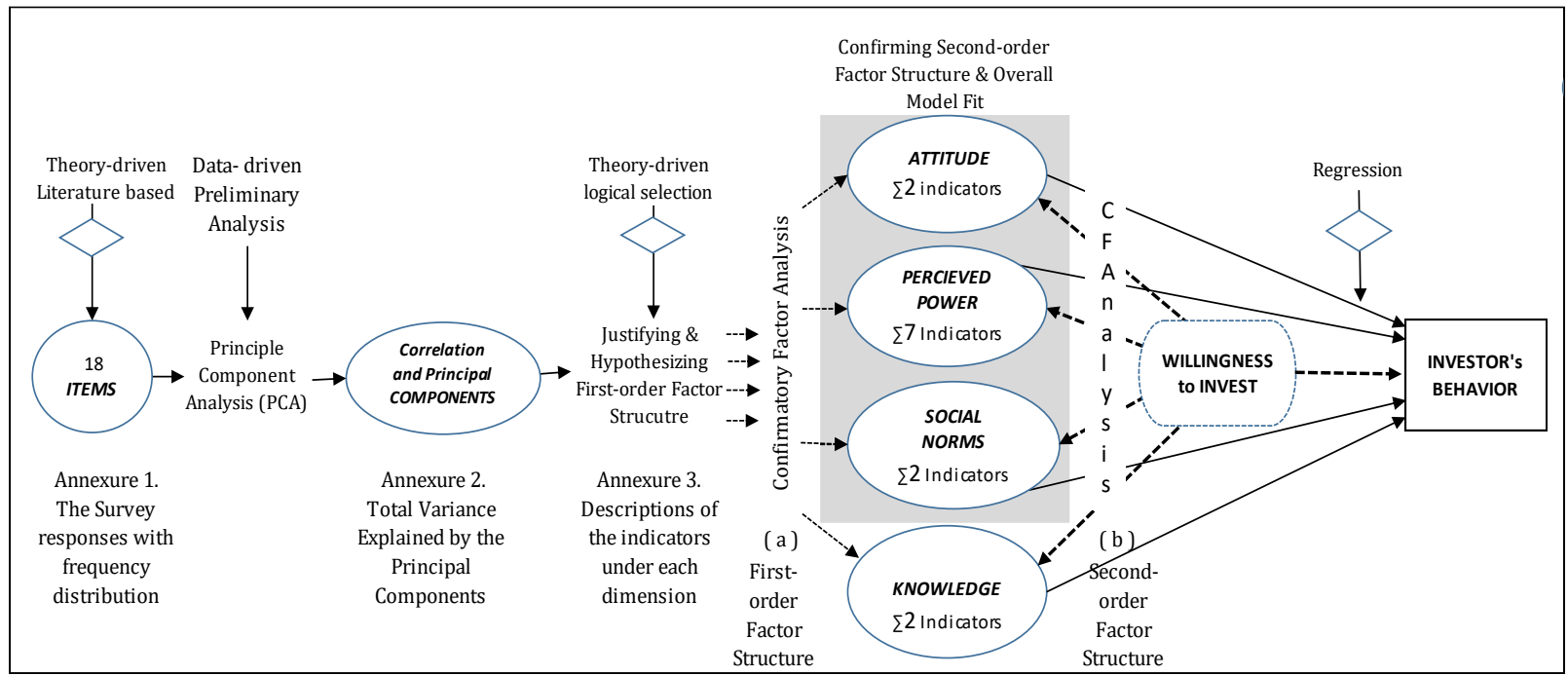

Fig. (1). The theoretical framework and hypothesized pathway analysis.

and Lee 2006). Studies have shown that self-efficacy is a clearer construct and depicts a better correlation with intention than perceived behavioral control (Armitage and Conner 2001). Therefore, in this research, power has been used in place of self-efficacy, as this type of efficacy fulfils the purpose of the present study. Power can be defined as the belief in one's capability to achieve certain financial goals (Forbes and Kara 2010).

\section{MATERIALS AND METHODS}

The methodology design we used here involved four stages, first to design a survey questionnaire based on behavioral theories and our understanding of the Fiji context. Then we did several model fit tests with two Confirmatory Factor Analysis (CFA) to construct the investors' willingness (IW) dimension. Third, we converted the hypothesized four dimension (attitudes, perceived power, financial knowledge and social norms) into a composite variables by adding constituent items of each dimension. Finally, regression analysis was done between the four hypothesized dimensions and the investment behaviour (IB). In other words, theoretically, first we found the underpinning influencing factors. Then, using Principal Component Analysis (PCA), we ascertained and hypothesized the four latent constructs (dimensions). After this, we found the higher-level mediating construct; IW, and converted the four hypothesized dimensions into variables (Wirth 1998; Schurz 2017), and finally, we established the nature of association between the four hypothesized dimensions and the IB using regression. Figure 1 illustrates this process.

We used the questionnaire to gather knowledge and behaviour of the people in relation to the SPX in several respects using 18 items. Five items concerned demographic factors: Age, Assets, Level of Education, Income level and Origin (Indigenous, Fiji-Indian and Other). Thirteen items had Likert scale answers: Strongly agree, Agree, Uncertain/NA, Disagree and Strongly disagree. There were 162 participants with university education. Two research assistants were involved in collecting data. The survey was carried out with employed university students majoring in Banking and Fi- nance. The frequency distribution of the responses is given in Annexure 1, Table B: Frequency distribution.

We initially formulated 18 items and their construct reliability was assessed using Cronbach's alpha (range $>0.7$ ) (Hair et al., 1998; Hair et al., 2011), and this resulted in 0.784 (as shown in Table A: Reliability test, in Appendix 1). Then we ran a PCA which is a variable-reduction technique in SPSS, and the results produced six principal components covering $63.27 \%$ of the cumulative variance of the variance in the original variables (see Appendix 1 Table C: Total Variance Explained). Based on the behavioral theories, knowledge of the Fiji context, together with the level of contributions of each factor and correlations and component matrices among all items, we identified four substantively important and empirically powerful dimensions and constituent items (Appendix 1-Table D: Component Matrix). For further analysis, based on CFA results, we computed composite measures for each factor by adding constituent items. For example, the composite measure for attitude was created by adding indicators F1 and F2. As shown in Figure 1, this was a process combining theories, local knowledge and, empirical findings (data). We tested: a) first-order Confirmatory Factor Analysis and b) a second-order CFA in the Structural Equation Modeling (SEM) framework (Bollen, 1989). After confirming the mode fit, we ran the regression between the hypothesized dimensions and the actual investments of the respondents in the SPX.

Each variable is represented by one item, as indicated in Table A, in Appendix 1. The responses to F1 and F2 were used as indicators of Attitude, F3-F9 (seven items) were used as indicators of Perceived Power, while F10 and F11 and F12 and F13 were used as indicators of Social Norms and Knowledge dimensions respectively.

\section{HYPOTHESIS AND THE MODEL}

To hypothesize the drivers of investing behavior, we first identify possible constructs mainly using TPB and extract the components which account for most of the variance in the original variables, using PCA. We tested two CFA models in a Structural Equation Modeling (SEM) framework to 


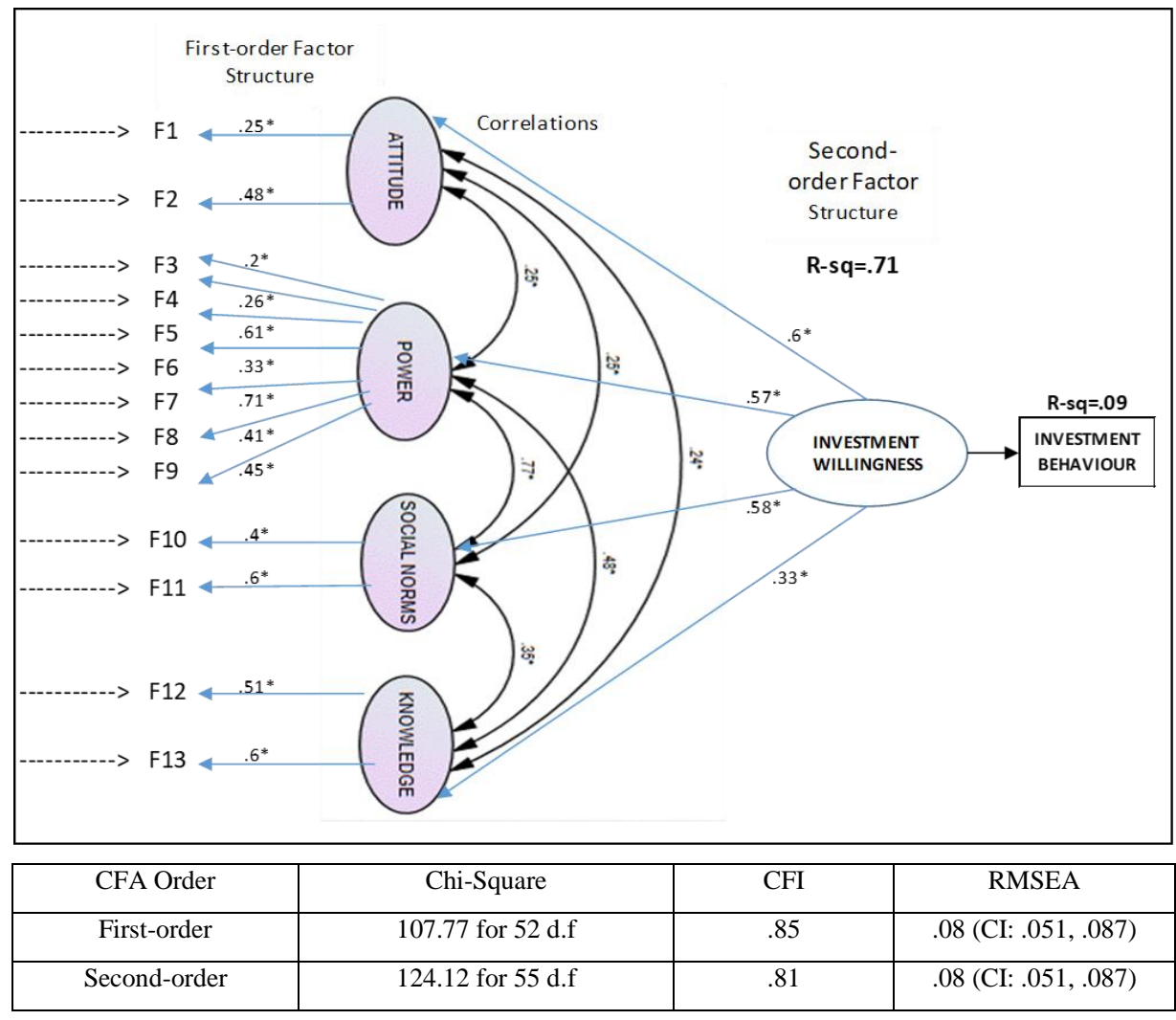

Fig. (2). Latent First-order and Second-order Factor Structure Reflecting 13 Factors, Four Dimensions of Investors' Willingness (IW) and their Correlations (measurement errors are not shown) Associated with the Investors' Behavior (IB).

analyze the survey data. We evaluated these models using several fit indices. The first CFA model included four correlated latent factors defined by 13 indicators (Factors) reflecting four hypothesized dimensions. The four latent hypothesized dimensions were correlated significantly. The second CFA model included second-order multi-level constructs reflecting respondents' willingness to invest in the SPX along with four constituent dimensions. The four dimensional factors showed significant and substantial loadings towards higher-order constructs of IW; the mediating driver towards IB. The model fit indices showed that this secondorder CFA model has an acceptable model fit. These results confirm that the four hypothesized dimensions-Attitudes (towards willingness), Perceived Power, Social Norms, and Financial Knowledge - are identifiable and distinct aspects.

The four dimensions were defined by 13 factors, based on the behavioral theories, and knowledge of the Fijian context. Then, together with the level of contribution of each factor and correlations and component matrices amongst all items we identified four substantively important and empirically powerful factors and constituent items. Statistically, latent factors reflect the common variance of indicators, and the squared loading of indicators reflects the amount of variance of the indicators explained by the latent factor. We assessed the distinctiveness or discriminant validity of the four hypothesized dimensions and the inter-dimension association. Second, we tested four second-order factor structures with second-level overall factors of investment intention. The second-level latent factor of investment intention was defined by the four first-level latent factors reflecting four dimensions of investment intention (see Fig. 1). The overall
IW variable was assessed by adding weighted loadings of four previously hypothesized dimensions; Attitudes, Power, Social Norms and Knowledge and the respective constructs of each dimension. Then the IW was regressed with IN found in significant correlations.

This analysis was performed with Mplus version 7.0 (Muthén and Muthén 2017) with Maximum Likelihood Estimation. A range of fit indices were used to evaluate the model fit of the models, including the chi-square statistic, Cumulative Fit Indices (CFI), and Root Mean Square Error of Approximation (RMSEA). For the chi-square fit statistic, the model is thought to fit the data well when the chi-square divided by the degrees of freedom is below 3.0 (Carmines and McIver 1981). The CFI and RMSEA are used to evaluate the models' fit due to the fact that they are not directly related to the sample size. We used the chi-square statistic, the CFI, and the RMSEA to evaluate the model fit. $\mathrm{Hu}$ and Bentler (1999) report that a CFI value greater than >.90 ensures that the model is not mis-specified. MacCallum, Browne, and Sugawara (1996) report that a RMSEA nearing .08 indicates a reasonably good model fit.

In sum, we expected to confirm the distinctiveness of the four identified distinct dimensions and their contributions to the overall IW based on their correlation matrix.

\section{RESULTS}

The first-order CFA model with four latent factors representing Attitudes, Power, Financial Knowledge, and Social Norm dimensions and the second-order factor structure with the overall IW factor is presented in Fig. (2). 
Table 1. Regression Results: The Four Dimensions on IB

\begin{tabular}{|c|c|c|c|c|c|}
\hline & $\begin{array}{c}\text { Standardized Coefficients }^{a} \\
\text { Beta }\end{array}$ & $\mathbf{t}$ & $p$ & \multicolumn{2}{|c|}{ 95.0\% Confidence Interval for B } \\
\hline (Constant) & & 7.283 & 0.000 & 1.697 & 2.96 \\
\hline Attitude & 0.135 & 1.580 & 0.116 & -0.012 & 0.109 \\
\hline Power & $-0.246^{* *}$ & -2.595 & 0.010 & -0.062 & -0.01 \\
\hline Knowledge & 0.041 & 0.489 & 0.625 & -0.044 & 0.072 \\
\hline
\end{tabular}

a. Dependent Variable: IB: Investment Behavior.

b. Note: $* \mathrm{p}<0.05$ and $* * \mathrm{p}<0.01$, Significant at the 5 and 1 percent levels respectively

c. $\mathrm{R} 2=0.089$

The first-order CFA model reflected an acceptable model fit (chi-square $/ \mathrm{df}=2.07, \mathrm{CFI}=.85, \mathrm{RMSEA} .08, \mathrm{CI}: .051$, .087). Overall, the CFA model provided evidence for a hypothesized first-order factor structure. Accordingly, Attitude, Power, Social Norms and Knowledge are four distinct dimensions of IW. The highest correlation between Power and Social Norms suggests a close relationship or the least distinctiveness between these two dimensions. The lowest correlations, between Attitude and Power, and Attitude and Knowledge suggest a weak relationship or the greatest distinctiveness between the two dimensions. However, significant correlations among the four factors suggest that there is a common variance across these factors, which may represent a higher-order factor. Thus, we tested the second CFA after incorporating the higher-order factor of investment willingness.

All 13 items showed significant substantial factor loadings to respective dimensional factors ranging from .2 to $.71(\mathrm{p}$ $<.05)$ showing the reliability and validity of these items in relation to the respective factor (Bollen, 1989). There were no significant cross-factor loadings. Four dimensional factors showed significant and substantial loadings $(.6, .57, .58$ and .33 to Attitude, Power, Social Norms and knowledge, respectively) to the second-order overall latent factor of the IW (significant error correlations were freed to be correlated, not shown in the figure).

The second-order CFA model reflected an acceptable model fit (chi-square/d.f $=2.26, \mathrm{CFI}=.81$, RMSEA $.08, \mathrm{CI}$ : .051 , .087). Overall, the CFA model provided evidence for a hypothesized factor structure. Accordingly, Attitude, Power, Social Norms and Knowledge are four distinct dimensions of investment intention. Also, there exists an overall, higherorder factor of investment intention along with four firstorder factors reflecting four dimensions.

Overall, it appears that these observed associations are specific to the Fiji socioeconomic context. All the variables explained $71 \%$ (R-squared) of variance in IW, and $9 \%$ variance in IB.

For further analysis, based on CFA results, we computed composite measures for each factor by adding constituent items. Table 1 shows the results of the regression of the IB and the predictors: Attitude, Power, Social Norms and Knowledge. The results showed that Perceived Power has a significant negative influence on IB $(\beta=-0.246, p<0.01)$ suggesting that, for every one unit increase in Perceived Power, there was a 0.245 units decrease in IB. Social norms also showed an influence on IB $(\beta=0.159, \mathrm{p}<0.05)$ suggesting that every one unit increase in the Social Norms percentage has resulted in a rise in IB by 0.159 units, after taking the influences of factors reflecting dimensions of Attitude and Knowledge into account. Attitudes and Knowledge showed no significant influence on IB directly. Overall, it appears that these observed associations are specific to the Fiji socioeconomic context. All the variables explained $8.9 \%$ (R-squared) of variance in IB.

\section{DISCUSSION AND CONCLUSIONS}

This framework attaches very close importance to the Attitude, the Social Norms and the Power dimensions with loadings of $.6, .57$ and .58 respectively. The Attitude dimension consists of the perceived importance and awareness of two factors (risk-seeker and not interested) and shows a loading of .6 towards IW. Moreover, these results are confirmed by the regression results, showing the least significance and the lowest coefficient for the association between knowledge and IB.

This study had two main objectives. First, to design and confirm the hypothesized theoretical framework with different dimensions of investment willingness towards investment intention and then for actual investment, and then to test this model in the Fijian context. In general, the results of the study supported the hypothesized framework for investment willingness and the four dimensions as influential factors for IW. Overall, the study provided useful findings about the varied severity of different dimensions, which may constitute valuable input for financial policy and programme planners.

This study revealed that four observed factors (Attitude, Power, Social Norms and Knowledge) are distinct dimensions of investment behavior. In addition, there exists a higher-level investment willingness factor which can also identified as intention to invest in the SPX.

The present study has used the Confirmatory Factor Analysis (CFA) in Structural Equation Modeling (SEM) framework to analyze the data. SEM allowed us to account for the measurement errors of the responses. Also, we have used several fit indices to evaluate the hypothesized model. This has enhanced the quality of estimated parameters and provided 
statistically more convincing results (Bollen, 1989). Further, second-order CFA confirm multi-level constructs (reflecting overall investment willingness and four constituent dimensions) are provided that can be considered for policy and programme formulation. We believe that this methodological framework can also be used to discover the influential factors of investment intention and then estimate the predictors of stock market investments in other developing countries with similar socioeconomic contexts.

The present study has several limitations. First, the sample size is relatively small; it would need to be larger to yield more statistical power. Second, respondents with more diverse backgrounds and from different geographical areas would have increased the generalizability of the study findings. Third, greater numbers of questionnaire items would have produced higher reliability of the factors reflecting different dimensions. Future studies should test this theoretical framework with a larger and more diverse sample, and with a more comprehensive instrument.

Despite these limitations, the current study enhances the existing knowledge about the assessment of individual investor's willingness and its driving forces in the Fijian context.

\section{CONFLICT OF INTEREST STATEMENT}

The authors declare that they have no conflict of interest.

Appendix 1.

\begin{tabular}{|c|c|}
\hline \multicolumn{2}{|c|}{ Table A: Reliability Statistics } \\
\hline Cronbach's Alpha & N of Items \\
\hline 0.784 & 18 \\
\hline
\end{tabular}

Table B. Frequency distribution.

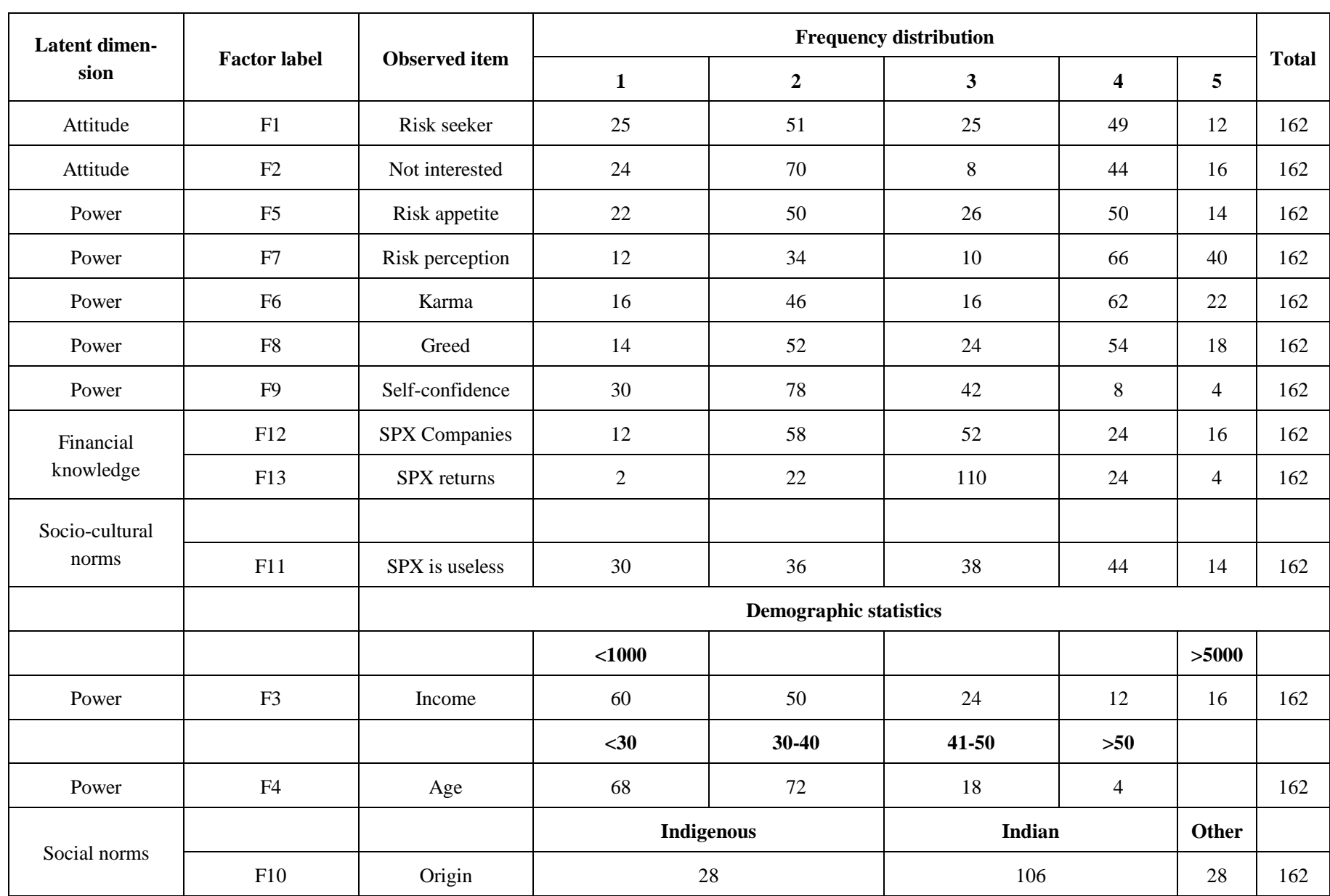

Table C. Total Variance Explained.

\begin{tabular}{|c|c|c|c|c|c|c|}
\hline \multirow{2}{*}{ Component } & \multicolumn{3}{|c|}{ Initial Eigenvalues } & \multicolumn{3}{c|}{ Extraction Sums of Squared Loadings } \\
\cline { 2 - 7 } & Total & \% of Variance & Cumulative \% & Total & \% of Variance & Cumulative \% \\
\hline 1 & 3.290 & 19.353 & 19.353 & 3.290 & 19.353 & 19.353 \\
\hline
\end{tabular}




\begin{tabular}{|c|c|c|c|c|c|c|}
\hline 2 & 1.945 & 11.439 & 30.792 & 1.945 & 11.439 & 30.792 \\
\hline 3 & 1.703 & 10.018 & 40.809 & 1.703 & 10.018 & 40.809 \\
\hline 4 & 1.519 & 8.937 & 49.747 & 1.519 & 8.937 & 49.747 \\
\hline 5 & 1.196 & 7.033 & 56.779 & 1.196 & 7.033 & 56.779 \\
\hline 6 & 1.103 & 6.488 & 63.268 & 1.103 & 6.488 & 63.268 \\
\hline 7 & 0.939 & 5.521 & 68.789 & & & \\
\hline 8 & 0.876 & 5.155 & 73.944 & & & \\
\hline 9 & 0.845 & 4.971 & 78.915 & & & \\
\hline 10 & 0.715 & 4.204 & 83.119 & & & \\
\hline 11 & 0.671 & 3.945 & 87.064 & & & \\
\hline 12 & 0.496 & 2.919 & 89.982 & & & \\
\hline 13 & 0.458 & 2.692 & 92.675 & & & \\
\hline 14 & 0.424 & 2.496 & 95.170 & & & \\
\hline 15 & 0.329 & 1.937 & 97.107 & & & \\
\hline 16 & 0.264 & 1.555 & 98.662 & & & \\
\hline 17 & 0.227 & 1.338 & 100.000 & & & \\
\hline
\end{tabular}

Extraction Method: Principal Component Analysis.

Table D. Component Matrix.

\begin{tabular}{|c|c|c|c|c|c|c|}
\hline \multirow{2}{*}{ Variable } & \multicolumn{6}{|c|}{ Component } \\
\hline & Power & Knowledge & Social & Attitude & 5 & 6 \\
\hline Income & 0.529 & -0.482 & 0.5 & -0.103 & 0.079 & 0.109 \\
\hline Age & 0.568 & -0.116 & 0.48 & -0.332 & -0.118 & -0.099 \\
\hline Assets & 0.612 & -0.544 & 0.276 & -0.126 & 0.068 & -0.095 \\
\hline Origin & -0.18 & 0.401 & 0.547 & 0.037 & -0.131 & 0.217 \\
\hline SPX & -0.299 & 0.33 & 0.223 & 0.237 & 0.285 & -0.094 \\
\hline SPX Co.1 & 0.391 & 0.451 & 0.085 & -0.328 & -0.143 & 0.253 \\
\hline Trading2 & 0.477 & 0.389 & -0.003 & -0.24 & 0.47 & -0.031 \\
\hline Risky3 & 0.296 & 0.185 & 0.473 & 0.456 & 0.293 & -0.275 \\
\hline Peers4 & 0.112 & 0.557 & 0.226 & 0.075 & -0.386 & -0.357 \\
\hline Averse5 & 0.566 & 0.366 & -0.176 & -0.098 & 0.035 & -0.429 \\
\hline Self Cf6 & -0.005 & 0.146 & 0.079 & 0.619 & 0.303 & 0.192 \\
\hline Karma7 & 0.445 & -0.096 & -0.273 & 0.308 & -0.383 & -0.136 \\
\hline Riskyii8 & 0.629 & 0.205 & -0.258 & 0.154 & 0.212 & 0.196 \\
\hline No Greed9 & 0.493 & -0.109 & -0.041 & 0.51 & -0.319 & 0.391 \\
\hline Karmab10 & 0.519 & 0.173 & -0.184 & -0.092 & 0.198 & 0.432 \\
\hline Lazy11 & 0.484 & 0.017 & -0.099 & 0.335 & -0.296 & -0.155 \\
\hline SPX Return12 & 0.128 & 0.448 & -0.174 & -0.289 & -0.217 & 0.189 \\
\hline Useless 13 & 0.303 & -0.16 & -0.556 & -0.021 & 0.257 & -0.263 \\
\hline
\end{tabular}

Extraction Method: Principal Component Analysis. 


\section{REFERENCES}

Akhtar, F., \& Das, N., 2019. Predictors of investment intention in Indian stock markets. International Journal of Bank Marketing, 37, 97113. doi:10.1108/IJBM-08-2017-0167

Ajzen, I., 1991. The theory of planned behavior. Organizational Behavior and Human Decision Processes, 50, 179-211.

Armitage, C.J. \& Conner, M.,2001. Efficacy of the theory of planned behaviour: a meta-analytic review. British Journal of Social Psychology, 40, 471-499.

Benjamin, D. J., Brown, S. A. \& Shapiro, J. M., 2006. Who is 'Behavioral'? Cognitive Ability and Anomalous Preferences. https://ssrn.com/abstract=675264 http://dx.doi.org/10.2139/ssrn.675264

Bollen, K. A.,1989. Structural Equations with Latent Variables. Wiley, New York.

Carmines, E. G. \& McIver, J. P. (1981). Analyzing Models with Unobserved Variables: Analysis of Covariance Structures. In Social Measurement: Current Issues, edited by G. W. Bohrnstedt and E. F. Borgatta, 65-115. Sage, Beverly Hills.

Cho, J. \& Lee, J., 2006. An Integrated Model of Risk and Risk-reducing Strategies. Journal of Business Research, 59, 112-20.

Cocco, J. F. \& Gomes, F. J., 2012. Longevity Risk, Retirement Savings, and Financial Innovation. Journal of Financial Economics, 103, 507$29 . \quad \mathrm{https} / / /$ econpapers.repec.org/article/eejfinec/ v_3a103_3ay_3a2012_3ai_3a3_3ap_3a507-529.htm.

Forbes, J. \& Kara, S.M., 2010. Confidence mediates how investment knowledge influences investing self-efficacy. Journal of Economic Psychology, 31, 435-443.

Gumbo, M. \& Sandada, M., 2018. The Determinants of Stock Market Participation: Evidence from. ACTA UNIVERSITATIS DANUBIUS, $14,642-654$. http://journals.univdanubius.ro/index.php/oeconomica/article/viewFile/4662/4533

Hair, J.F., Ringle, C.M. \& Sarstedt, M., 2011, "PLS-SEM: indeed a silver bullet", Journal of Marketing Theory and Practice, 19, 139-152.
Hair, J.F., Black, W.C., Babin, B.J., Anderson, R.E. \& Tatham, R.L., 1998, Multivariate Data Analysis, Upper Saddle River, Prentice Hall Inc, New Jersey.

Hu, L. T. \& Bentler, P. M., 1999. Cutoff criteria for fit indexes in covariance structure analysis: Conventional criteria versus new alternatives. Structural Equation Modeling: A Multidisciplinary Journal, 6(1), 1-55. doi.org/10.1080/10705519909540118

Khan, S. N., 2016. Impact of Financial Literacy, Financial Knowledge, Moderating Role of Risk Perception on Investment Decision. SSRN Electronic Journal. http://doi: 10.2139/ssrn.2727890.

Korniotis, G. M. \& Kumar, A., 2011. Do Older Investors Make Better Investment Decisions? The Review of Economics and Statistics, 93, 244-65.

MacCallum, R. M., Browne, M. W. \& Sugawara, H., 1996. Power Analysis and Determination of Sample Size for Covariance Structure Modeling. Psychological Methods 1, 130-49.

Makarov, D. \& Schornick. A. V., 2010. Explaining Households' Investment Behavior. INSEAD Working Paper No. 2010/44/FIN, 5-30.

Muthén, L. K. \& Muthén, B. O., 2017. Mplus User's Guide (7th ed). Author, Los Angeles.

Saliya, C. A., 2020. Stock Market Development and Nexus of Market Liquidity. International Journal of Finance and Economics, doi.org/10.1002/ijfe.2376.

Schurz, G., 201. Patterns of Abductive Inference. In Springer Handbook of Model-Based Science, edited by Lorenzo Magnani and Tommaso Bertolotti. Cham: Springer. http://doi:10.1007/978-3-319-305264_7.

Tauni, M. Z., Fang, H. X. \& Iqbal, A., 2017. The Role of Financial Advice and Word-of-Mouth Communication on the Association Between Investor Personality and Stock Trading Behavior: Evidence from Chinese Stock Market. Personality and Individual Differences 108, 55-65. http://doi: 10.1016/j.paid.2016.11.048.

Wirth, U., 1998. What is Abductive. Retrieved from https://core.ac.uk/download/pdf/14512821.pdf 